\title{
|||||||||||||||||||||||||||||||||||||||||||||||||||||||||||||||||.
}

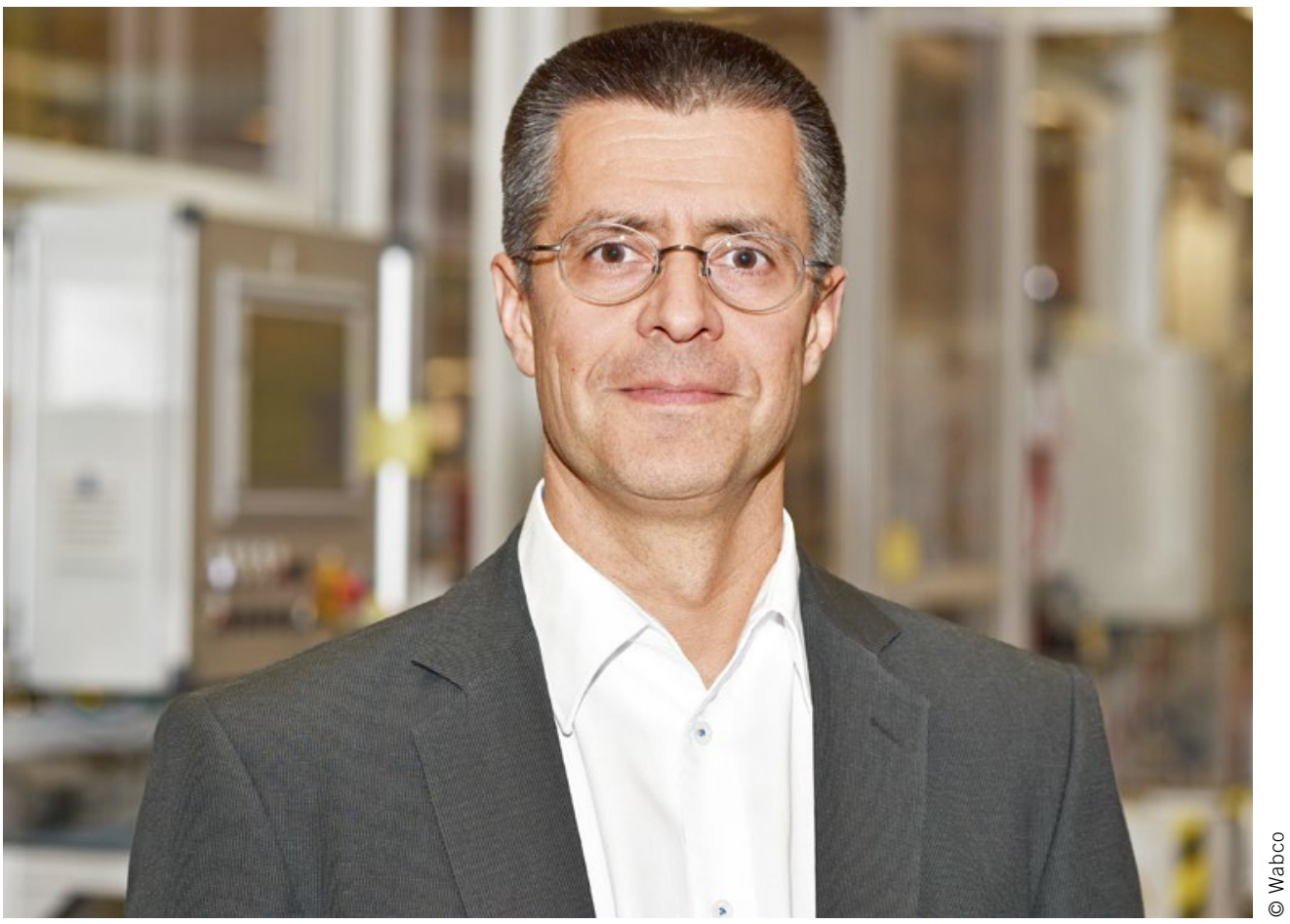

Dr.-Ing. Thomas Dieckmann

Leader of Advanced Development at Wabco, Member of the

ATZ Advisory Board

\section{The Sequence of Automation Levels}

SAE defines levels of autonomous driving, starting at level 1 leading up to level 5. At a first sight, it seems like these levels would logically build upon the other, as if this would be the order in which these levels will be introduced in the future. However, the question if this really will be the case is justified, as the different levels neither necessarily describe a step-bystep order nor present a single option of classifying. Truck platooning for example, a frequently discussed concept in the commercial vehicles industry in these days, is hard to be classified within one of the levels in its first stages. When distance control is automated, but the driver still controls lateral control of driving, this cannot be clearly assigned to one of the levels between 1 and 5. In this case, the OEM would be responsible for the longitudinal control while the driver has the lateral control.

When questioning the order sequence, the step from level 2 to higher levels of automation is of particular importance. Here, the responsibility for vehicle guidance is transferred from the driver to the OEMs or its system suppliers for the first time. For this reason, the OEMs involved need to think about which responsibilities they want to assume and whether this is economically justifiable. But even if they deliberately decide to keep the driver in the main responsibility and thus stick to level 2 , the driver's attention must be ensured and kept on the traffic. Otherwise the system unintendedly will become a level 3 system, in which the driver would have to take over the driving task again within a short period of time.

From this level 3 onwards, the question arises how OEM and system supplier will take over the responsibility of vehicle control that is transferred for the first time. Which experiences and which safety levels are needed? Shall systems be as good as today's average drivers or do you have to stick to higher goals? Failure scenarios must also be included. Maybe the step to level 3 is already so expensive that it does not pay off as an intermediate step and it will be strived for level 4 immediately. This step requires a further increase in sensor technology, since longer times without a driver and thus more complex situations need to be covered. All of this is very expensive and will bring additional challenges, especially in the commercial vehicle industry, since the trailer must also be considered alongside the truck. Therefore, level 4 is often seen to be realised in a distant future. Especially in the commercial vehicle sector the development towards higher levels has a strong business case, in contrast to the car industry. If the driver would be freed from driving on highways for example, this could potentially save several thousand euros per year. The retrofitting of the necessary sensors and control units could therefore be profitable.

It is possible that heavy commercial vehicles will become the pioneers of automation and thus drive important innovations. 